\title{
[POSTER] Augmented Reality Assistance in the Central Field-of-View Outperforms Peripheral Displays for Order Picking: Results from a Virtual Reality Simulation Study
}

\author{
Patrick Renner* \\ Thies Pfeiffer ${ }^{\dagger}$ \\ Cluster of Excellence Cognitive Interaction Technology \\ Bielefeld University
}

\begin{abstract}
One area in which glasses-based augmented reality (AR) is successfully applied in industry is order picking in logistics (pick-byvision). Here, the almost hands-free operation and the direct integration into the digital workflow provided by augmented reality glasses are direct advantages.

A common non-AR guidance technique for order picking is pickby-light. This is an efficient approach for single users and low numbers of alternative targets. AR glasses have the potential to overcome these limitations. However, making a grounded decision on the specific AR device and the particular guidance techniques to choose for a specific scenario is difficult, given the diversity of device characteristics and the lack of experience with smart glasses in industry at larger scale.

The contributions of the paper are twofold. First, we present a virtual reality (VR) simulation approach to ground design decisions for AR-based solutions and apply it to the scenario of order picking. Second, we present results from a simulator study with implemented simulations for monocular and binocular head-mounted displays and compared existing techniques for attention guiding with our own SWave approach and the integration of eye tracking.

Our results show clear benefits for the use of pick-by-vision compared to pick-by-light. In addition to that, we can show that binocular AR solutions outperform monocular ones in the attention guiding task.
\end{abstract}

Index Terms: H.5.2 [Information Interfaces and Presentation (e.g. HCI)]: User Interfaces-Miscellaneous

\section{INTRODUCTION}

Augmented reality (AR) glasses enable providing information in a mobile, hands-free way. They are already actively used in the areas of construction, maintenance or health care. One industrial application where the benefits of AR glasses are obvious is order picking. Here, AR approaches compete with classical guidance approaches like lights at the target locations (pick-by-light) which can efficiently guide single users to one of a small number of targets. The question addressed in this paper is how AR hardware and guidance techniques can be designed to increase efficiency in order picking.

Guiding the users' attention using AR glasses is not trivial, as objects of interest can be located outside the field of view (FOV) of the glasses (or even, e.g., behind the user). This way, it is often necessary to guide users to targets which do not overlap with the AR display or which are not visible at all. Moreover, the smaller the AR FOV, the more likely the user is to gaze at locations in the

\footnotetext{
*e-mail: prenner@techfak.uni-bielefeld.de

†e-mail: tpfeiffer@techfak.uni-bielefeld.de
}

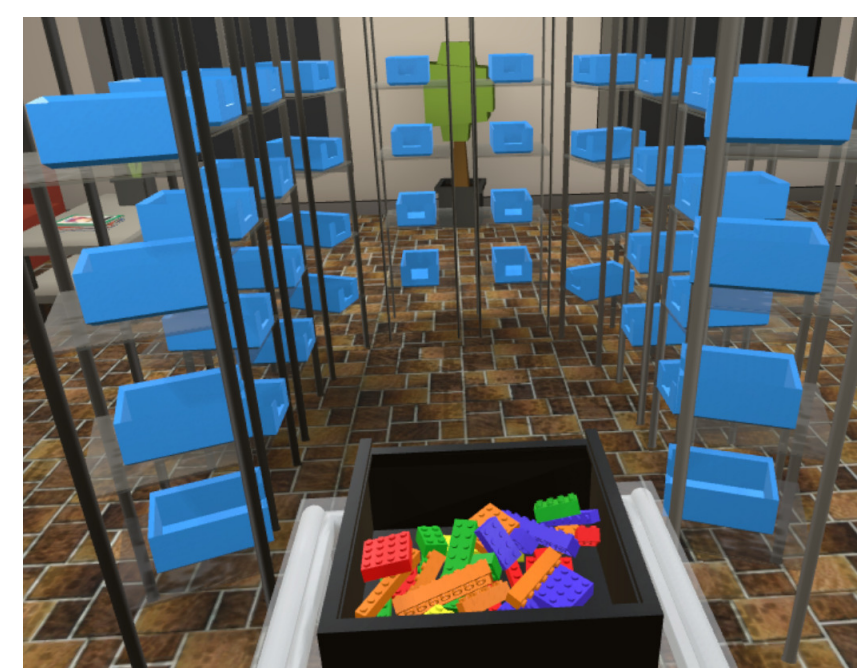

Figure 1: The scenario of the study: Participants pick parts from the black box and have to place them in the blue boxes located in the shelves.

environment where the AR display does not overlap with. In such off-AR-screen cases, it could be helpful to provide visual feedback on the AR display that can be perceived and interpreted using peripheral vision, so that the users' attention can remain on the real environment and the task at hand. Such peripheral visual feedback has been successfully tested using non-AR light-based approaches in simulated aviation [11]. Important feedback for order picking would be the direction and distance of the target and a confirmation when the correct target has been located. Mechanisms to make visual information perceivable in the periphery are high contrast changes or movements. To differentiate between off-screen and onscreen situations, eye tracking capabilities could be used to keep track of the users' attention in real time.

In a virtual reality simulation of a Lego-like brick placing scenario (see Figure 1), we evaluate three different aspects of AR solution design for attention guiding techniques: first, we compare peripheral monocular AR with binocular central FOV AR. We realize this by simulating device screens of a Google Glass and a Microsoft HoloLens. Second, we compare a non-AR baseline using pick-by-light with AR-based attention guiding by the display of an arrow or alternatively our own spherical waves (SWave). Third, we implement off-AR-display eye-gaze-aware visual feedback for peripheral vision by using eye tracking.

This paper is structured as follows: First, work about visual guiding techniques and the VR-based simulation is reviewed. Then the evaluated guiding techniques are explained, as well as the contrasted AR devices. Then the paper reports about the conducted study and the results, before closing with a summary. 


\section{Related Work}

A straight-forward AR-way of guiding the attention towards an item in the environment is highlighting [6]. In its basic form, the outlines of the target are emphasized using a 3D registered overlay. This technique is naturally constrained to the area of the AR display and thus only applicable when the user is already closing in towards the target, as the user has to move his head to look for the highlighted target by bringing the AR display into overlap with the real environment. In the same paper [6], Feiner at al. used a label within the AR-view and an associated line in the style of a dotted rubber band to connect to targets outside the AR-view.

A different approach to guide attention towards a target is by prompting the direction towards the target using an arrow. This has been described for pedestrian navigation [5] and for guiding tourists to points-of-interest [16]. It has also been shown to be efficient in the case of directing drivers of a car [20] using a 3D arrow. A fixedscreen $2 \mathrm{D}$ arrow has also been used to help mechanics to re-orient towards targets behind them [9].

In a non-AR context, Baudisch and Rosenholtz [2] propose the Halo technique for guiding attention to targets located outside a 2D screen: They surround off-screen targets with rings that reach into the border region of the screen. This way, not only the location is indicated, but also the distance (on a plane) can be approximated based on the size and curvature of the visible arc.

The omnidirectional attention funnel (OAF) is an animated visual guiding system, in which a flexible tunnel of frames is drawn from the current head position and orientation to the intended position and orientation when facing the target [3]. In a comparison study against audio cuing by naming and selection-box highlighting, the OAF was shown to improve search assistance performance in terms of shorter search times, lower errors, and a lower cognitive load.

In real-life conditions, a combination of several techniques might be adequate, e.g., as described in [9]. They use a 2D arrow for extreme angles in which the user is required to turn around, then switch to $3 \mathrm{D}$ arrows when the orientation change is less than $90^{\circ}$ and once the target is covered by the AR-view slowly migrate to a highlighting, which in turn is only active for five seconds.

While especially the latter combination of techniques appears to be very plausible and was well accepted by the participants in their study, to our knowledge there do not exist extensive reports comparing and covering the individual visualization strategies, even less covering combinations of designs. A better knowledge of the advantages and disadvantages of different visualizations, however, is necessary to support an adaptation of the system to user preferences and needs.

\subsubsection{Guiding Techniques to Support Order Picking}

Classically, order picking is supported using simple lists of the target objects. However, AR techniques have been shown to provide superior performance [18] and recently Guo et al. [7] showed that displaying the next item to pick on a head-up-display on a HMD or on a display mounted on the cart outperforms picking using lists or pick-by-light, where the place to pick is highlighted using an LED at its location.

Our work addresses one central question regarding order picking: How can a technical system guide the attention of a user towards a specific target object in the environment? In the domain of $\mathrm{AR}$, this research problem is one of attention management and augmentation in AR interfaces [3]. Attention guidance is relevant in many application areas, such as virtual teleconferences, visual search (e.g. order picking [17 13]), spatial navigation, or procedural queuing. The work at hand focuses on the guidance of the attention of a worker towards a particular picking/placing bin in a working environment.

\subsection{Design Space of Visual Attention Guidance}

AR glasses provide a huge and still largely unexplored design space. Some of the key aspects of this design space will be brought into focus in the following.

Firstly, AR glasses may present information either on the canvas of their display (in-view) or registered to the 3D position of the target object (in-situ). Combinations are also possible, e.g., by presenting the projected $3 \mathrm{D}$ position of the object on the $2 \mathrm{D}$ canvas of the display.

The display area of commercially available AR glasses covers a field of view of $20^{\circ}$ to $90^{\circ}$ [4]. In contrast, the full field of view of a human is about $180^{\circ}$. Hence, a large part of the natural field of view is not covered by the AR display. Thus in the past, visualization techniques have been optimized for small fields of view. However, Kishishita et al. [10] show that larger fields of view increase performance in visual search tasks. In addition to its size, the AR field of view may be located in the central field of view of the wearer (e.g. Microsoft HoloLens) or towards the periphery (e.g. Google Glass). In a maintenance task, Zheng et al. [22] found evidence that when showing in-view instructions, central FOV outperforms peripheral FOV in terms of task completion time. However, they did not evaluate in-situ visualizations.

As a direct consequence of the previous aspects, the area around the user of an augmented reality system may be classified into three regions. The AR-FOV is the region which is covered by the AR display. The FOV is the field of view of the user of the AR system. AR-FOV and FOV typically overlap, with the AR-FOV being a subregion of the FOV. The Off-FOV area is what the user cannot see without any head movement. Objects of interest might be visible in the FOV, but not necessarily be covered by the AR-FOV.

\subsection{Eye Tracking for AR Assistance}

By the integration of eye tracking into AR glasses, several problems can be addressed: first of all, the calibration of the AR glasses can be optimized, as the devices have to detect the position of the eyes and their orientation, which is relevant for creating the correct visual perspective. Second, eye gaze might be used as an input modality for interaction with the device. Finally, the AR glasses may monitor the direction of gaze and modulate their feedback accordingly.

\subsection{VR Simulations for the Prototyping of AR Systems}

When testing AR systems, there are many technical aspects that make reliable studies difficult. First, in particular with optical seethrough systems, there is the problem of maintaining constant environmental conditions, e.g. lighting, for each participant. Second, if repetitions of manual interaction tasks with randomized placements are required, it takes time to re-arrange parts between tasks without the participant watching. This increases overall study-time and raises chances for human errors. Third, when using typical visionbased AR systems there is always the possibility of tracking errors, latencies, or other technical problems resulting in perceptual errors. Some of these errors might not be noticed by the experimenter. All of these errors, however, will influence the outcome of the study. Fourth, the general performance of the available hardware will also influence the outcome, e.g. a high tracking or display latency will severely affect the evaluation of the displayed information.

To tackle these issues, we developed a system which can abstract from some hardware and environmental factors. It is capable of simulating AR devices in VR. The 'real world' content is realized like a normal VR simulation, but additionally AR hardware is also simulated. This makes it possible to evaluate AR techniques for arbitrary devices and completely independent of changes in the environment. Different fields of view (FOVs), tracking latencies etc. can also be simulated and thus be systematically tested. 
Wafaa et al. [21] were among the first to present the idea and discuss an architecture for a VR-based simulation of AR on a conceptual level. Early other approaches remained on a very technical level and simple artificial scenarios [12]. Recently, Steindecker et al. [19] took up this idea but focused on the design of the optics of an AR system. Alce et al. [1] presented a similar approach. However, they focus on an AR system that allows only for interactions in a stationary situation.

\section{A Study on Guiding Techniques and AR Hardware}

In previous studies $([14],[15])$ we showed that the arrow-based guiding technique and our newly proposed SWaves method based on moving waves on a sphere are both promising candidates for pick-by-vision tasks. In this study, we now include eye tracking to modulate the feedback based on the user's attention.

Two different AR devices are simulated. As peripheral FOV AR device, Google Glass with a FOV of 18 degrees and the display positioned at the periphery of the right eye was simulated. As central FOV AR device, a Microsoft HoloLens was simulated with approximately 35 degrees FOV. For both devices we added a condition with attention based feedback modulation.

\subsection{Pick-by-Light}

For being able to compare the AR-based techniques to a classical guiding approach, guiding using a picking light was chosen (Figure 2 a). The idea is to have a lamp attached to all possible targets. Only the lamp of the currently relevant target is switched on, while all other lamps are switched off.

\subsection{Arrow-Based Guidance}

An in-view arrow is rotated around the center of the AR display such that it points to the target object (Figure 2p). When the target object overlaps with the AR display, an additional 3D highlight is shown at the location of the target making sure that objects of different depth can be disambiguated (Figure $2 \mathrm{~d}$ ).

In addition to that, a distance measure is integrated into the guiding technique: Using eye tracking, the transparent background color of the AR display slowly changes from red to yellow depending on how close the user looks to the direction of the target. When the user is directly fixating the target, the color changes to green. If eye tracking is not available, head orientation is used instead of eye gaze direction.

\subsection{Spherical Wave-Based Guidance}

As originally introduced in [14], we developed a new guiding technique called Spherical Waves ("SWave") which is inspired by the Halo technique [2] for 2D off-screen visualization. The Halo technique tries to make the user aware of a point of interest (POI) that lays beyond the borders of the display by imagining a circle around the point of interest which intersects with the display in such a way that a fraction of the circle, an arc, is drawn in the visible area.

The Halo technique focuses on supporting the navigation to 2D digital content which can only be viewed on the available display. So there are basically two conditions: either the POI is on-screen, so that the user can see it, or it is off-screen and the user cannot see it. In AR, we have three conditions: either the AR display overlaps with the POI and thus the user can see both, POI and the visualization for guiding the attention towards the POI (e.g. Halo). Second, the POI may be off-screen regarding the AR display but still in view of the user. Third, the POI might be beyond the field of view of the AR display and the current field of view of the user. With our design of the SWave guiding technique we in particular want to address this intermediate situation with the POI being present in the field of view of the user but beyond the coverage of the AR display. The graphical design shall enable the user to gaze off-screen towards the target, but still be able to extract relevant information
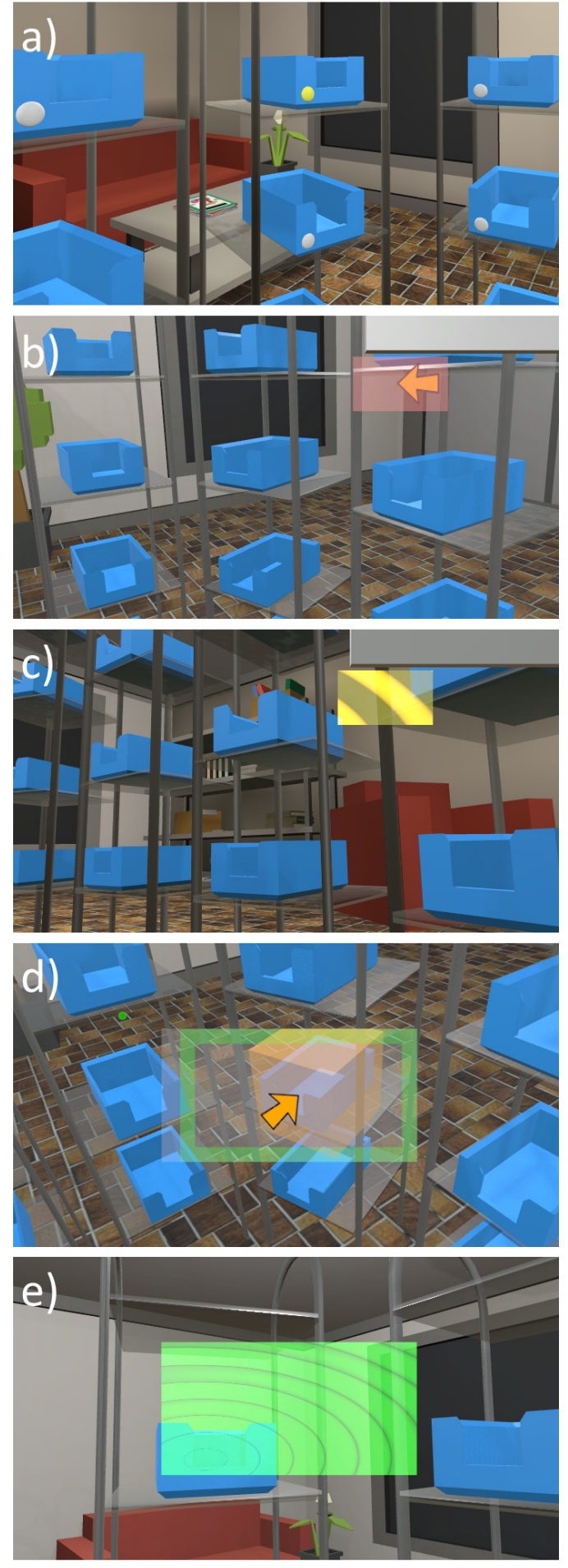

Figure 2: The different combinations of guidance techniques and AR devices: a) A picking light switched on at the target box. b) An arrow shown in the AR display of Google Glass. The background color is red as the user is looking far away from the target location. c) DynSWave applied on the Google Glass. The background color of the waves is yellow as the user is looking close to the target location. d) The arrow on the Microsoft HoloLens. The user is looking at the correct position, an additional highlight is shown and the former green background color turns into a frame to not occlude the target. e) DynSWave applied on the HoloLens. The color of the waves is green as the user is looking at the correct location. 
about direction and distance towards the target using peripheral vision. The spherical waves are thus created to have a high contrast and in addition to the original Halo design, the waves move towards the target to support the perception of movements towards the target. This is realized by creating a sphere which is centered at the head position of the user with a radius which is permanently updated to match the distance to the target (Figure 2k). On this sphere, the waves are then rendered using a GPU shader. The advantage of this guiding technique is that it serves as an in-situ visualization by having the waves converging at the $3 \mathrm{D}$ target position but also has in-view aspects as the waves are always visible and thus providing information about the direction to the location of the target by their curvature and direction of movement.

In the attention modulated feedback condition, the speed of the spherical waves was adjusted by the angle between the current gaze direction and the target location: The waves move fast when the target is behind the user (with a maximum of six times the base speed of approximately $5 \%$ s) and completely stop when it is directly fixated. In addition to changing the speed, changing background color of the AR display as described for the arrow-based guidance was used (Figure 2e). We call the extended SWave "DynSWave".

\section{Methodology}

In an order placement scenario, we conducted a within-subject experiment evaluating seven combinations of the arrow-based guidance and the DynSWave guidance with simulated Google Glass and Microsoft HoloLens as independent variables. The baseline condition was showing a picking light ("LIGHT"). Three conditions were applied on Google Glass: The arrow-based guidance with active eye tracking ("ARROW_ET") as well as the DynSWave guidance without ("DYNSWAVE") and with eye tracking ("DYNSWAVE_ET"). On the simulated Microsoft HoloLens, also three guidances were applied: The arrow-based guidance without eye tracking ("ARROW"), and both variants of DynSWave ("DYNSWAVE" and "DYNSWAVE_ET").

The evaluation of the benefits of feedback modulated by eye tracking was focused on the DynSWave technique, as the arrowbased technique was not in particular designed for peripheral vision. However, we still made use of the color-based peripheral feedback in the ARROW_ET condition, as we expected it to have some benefits there. We did not make an extensive testing of all conditions after realizing during pilots that the full set was too demanding for participants.

\subsection{Presentation and Interaction}

An HTC Vive HMD with an integrated $120 \mathrm{~Hz}$ binocular Pupil Labs eye tracker was used for simulating VR and AR content. With a latency of $5.7 \mathrm{~ms}$, the eye tracker is suitable for real-time interaction. Its gaze accuracy is $0.6^{\circ}$, the precision is $0.08^{\circ}$. Due to the HMD room-scale tracking capabilities of the HMD, study participants were able to move freely in their workspace. The two controllers of the HTC Vive were used to visualize simulated hands which made a grasping movement when pressing the trigger.

\subsection{Scenario}

A living-room-like environment was simulated to provide a familiar spatial reference: The user found himself in a room with a couch, a table and some plants. In the center, right in front of the user, there was a box filled with 40 Lego bricks located on a table. Around the user, ten shelves with four small boxes each were arranged (see Figure 11. Thus, there was one specific box for each brick.

\subsubsection{Task}

The task was to sort each of the 40 bricks into the correct boxes repeated for all different guiding techniques. Thus, in total $40 * 7=$
280 bricks had to be placed per participant. The sequence of guiding techniques was balanced using a Latin square design. For each trial, the permutation of the target boxes was chosen randomly. When the user grasped one of the bricks, the current guiding technique was enabled. When the brick was placed in one of the small boxes, a visual color feedback and a sound notified about correct or wrong placement and the placed brick disappeared to make sure that it was not possible to see which boxes were already used in subsequent placements.

Using order-placement instead of the actual order-picking scenario had two advantages. First, the task ends as soon as the target was found and the object is placed, thus at the moment when no guidance is needed anymore. Second, the user can decide when to start with the next part simply by picking it from the box.

\subsubsection{Procedure}

In the beginning, participants stood in front of the table with the box of Lego bricks. In order to get used to the environment, they were allowed to orient themselves and grasp some of the bricks until they felt comfortable with the simulation.

Before actually starting with the task, participants were given the possibility to do some test placements with the current guiding technique. Then, they were instructed to place the 40 bricks as accurate and fast as possible. After they finished a condition, participants were asked to fill out a Nasa TLX questionnaire [8].

\section{Results}

We had 21 participants ( 8 male and 13 female) taking part in our experiment. Their age ranged from 19 to 37, with a mean of 23.1 years (sd: 3.9). They were all students of our university from various fields of study. Only two of them had reasonable experience with virtual reality, while 10 were experienced with computer games. Objective data as time-on-task and head movements were acquired as well as subjective data from the NASA TLX questionnaire. The results were statistically tested using ANOVA and Tukey's honest significance test.

\subsection{Objective Results}

Making no use of AR guidance, pick-by-light lead to a mean time of 4.73 s to place one brick (sd: 0.93). All guidances applied on the simulated Google Glass performed worse: The slowest method was showing the arrow with a mean time of $6.51 \mathrm{~S}$ (sd: 2.02). With the DynSWave guidance placing a brick took on average $5.15 \mathrm{~s}$ (sd: 3.21) and with eye tracking $5.38 \mathrm{~s}$ (sd: 4.19). The guidances applied on the simulated Microsoft HoloLens led to better performance. Using the arrow, participants needed on average $4.85 \mathrm{~s}$ (sd: 1.45). The DynSWave guidances had an average of $4.05 \mathrm{~s}$ (sd: 1.77) without eye tracking and $4.09 \mathrm{~s}$ (sd: 1.09) with eye tracking. The difference of the arrow-based guidance applied on Google Glass to the DynSWave guidances applied on the Microsoft HoloLens was significant $(\mathrm{p}<0.05)$. Additionally, the DynSWave technique on HoloLens performed significantly better than the same guidance on the simulated Google Glass. For an overview over the times needed see Figure 3

Figure 4 shows that the different guidance techniques led to reduced head movement in comparison to showing the picking-light with which participants moved their head on average $481.2 \mathrm{deg}$ (sd: 42.1). Using the arrow guidance on the Google Glass reduced movements to a mean of $451.8 \mathrm{deg}$ (sd: 75.8), using the DynSWave guidance without eye tracking to $439 \mathrm{deg}$ (sd: 166.6) and using eye tracking to $424.5 \mathrm{deg}$ (sd: 130.5). Applied on the Microsoft HoloLens, the arrow guidance led to an average head movement of 387.5 deg (sd: 55). Using the DynSWave guidance, participants moved their head on average 382.4 deg (sd: 101.4) with added eye tracking $368.3 \mathrm{deg}$ (sd: 55.2). Hereby the pick-by-light technique performed significantly worse than all guidances applied on 


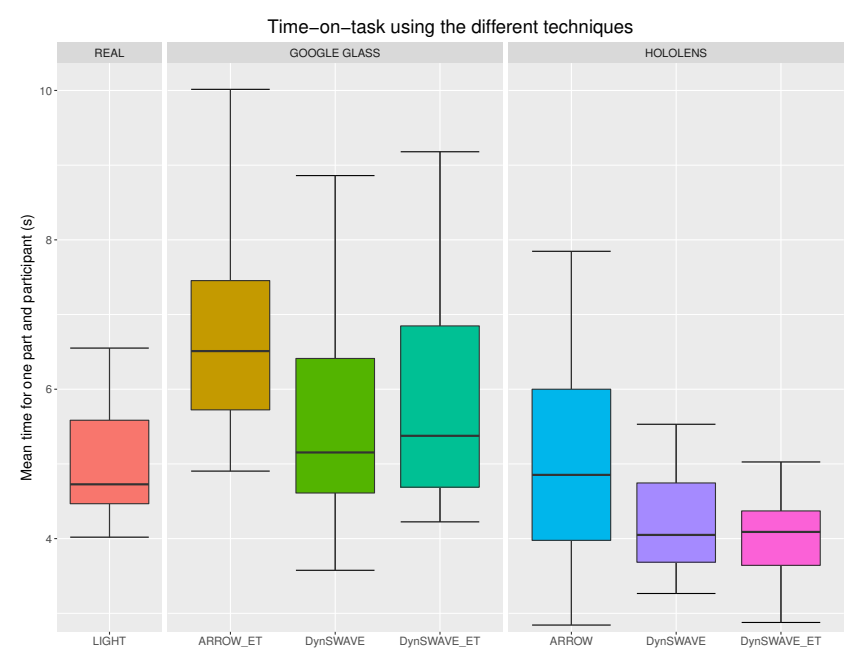

Figure 3: The mean time in seconds needed to place one brick.

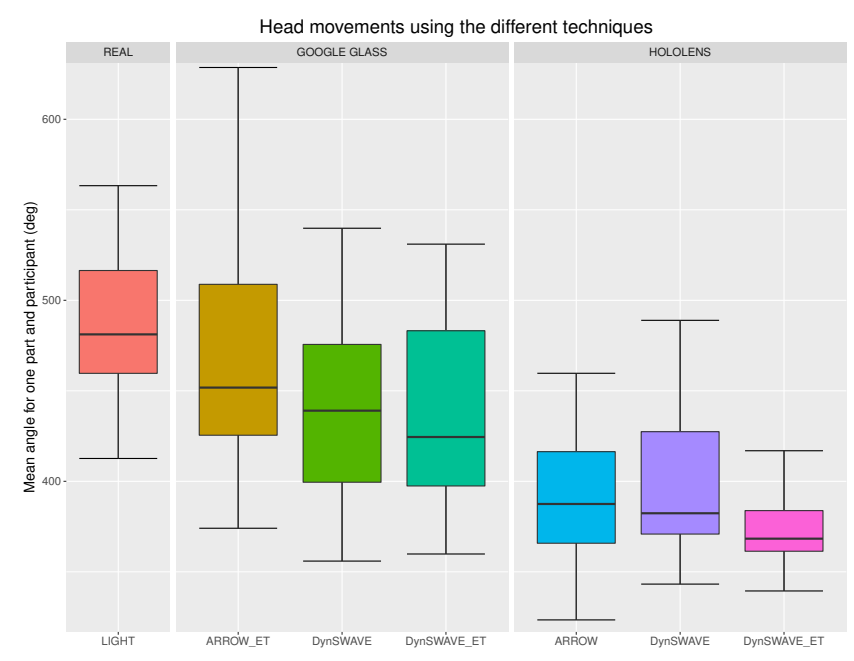

Figure 4: The mean head movement in degrees conducted to place one brick.

the HoloLens $(\mathrm{p}<0.001)$. All guidances applied to the HoloLens performed significantly better than the arrow guidance applied on Google Glass $(\mathrm{p}<0.001)$. The DynSWave guidance with eye tracking on the HoloLens led to significantly less head movements than all guidances on Google Glass $(\mathrm{p}<0.01)$ as well as pick-by-light $(\mathrm{p}<0.001)$.

\subsection{Subjective Results}

The results of the NASA TLX can be found in Figure 5 The guidances applied on Google Glass led to the highest subjective task load: Using the arrow, the raw score was on average 56 (sd: 22), using DynSWave without eye tracking 35 (sd: 25) and using DynSWave with eye tracking 41 (sd: 23). On the HoloLens, task load using the arrow had a raw score of 28 (sd: 17), using DynSWave without eye tracking lead to score of 20 (sd: 22) and with eye tracking to 25 (sd: 19). Both DynSWave guidances applied on the HoloLens were rated to lead to a significantly lower task load than the arrow guidance applied on Google Glass $(\mathrm{p}<0.05)$.

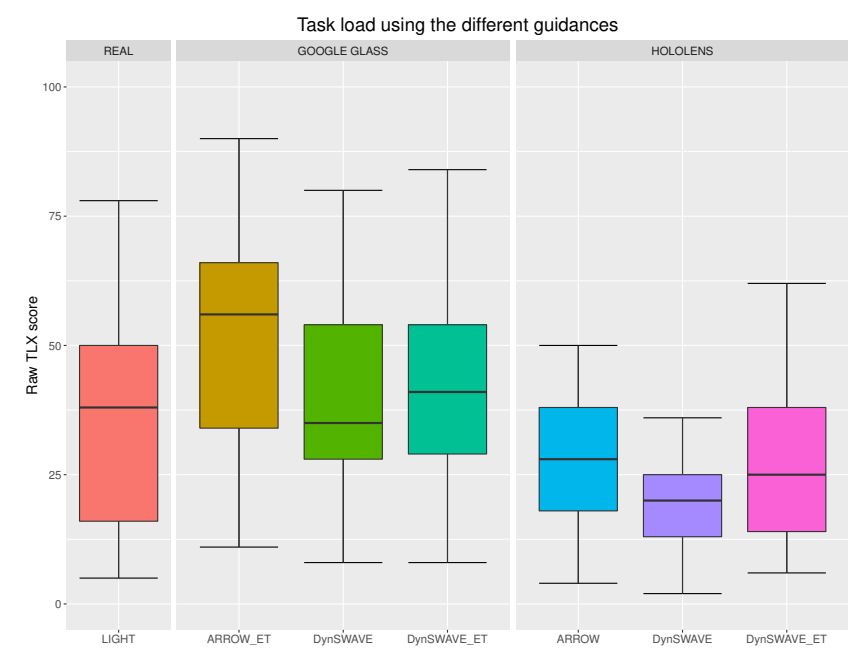

Figure 5: The subjective task load as rated using NASA TLX.

\subsection{Discussion}

Having an overall look at the results, one can see that the choice of AR device has a strong effect on performance. Regardless of the applied guidance technique, the Google Glass as monocular peripheral AR device resulted in the slowest task performances. Participants were faster using the non-AR picking-by-light, even if they had to conduct more head movements to find the correct target. We suppose it is most likely that the positioning of the AR display made it more difficult to follow the AR instructions. Also, the arrowbased guidance technique resulted in slower performance than the DynSWave technique, regardless of attentional modulation. Here we assume that the combination of in-situ and in-view elements provided by DynSWave provides more information which can be used by the participants to quickly find the target.

The results regarding speed basically coincide with the task load reported by the participants using the Nasa TLX. Task load using Google Glass was slightly higher than using the non-AR pickingby-light, using the HoloLens it was slightly lower. Looking at the pick-by-vision guidance techniques themselves, the DynSWave technique could reduce task load in comparison to the arrow-based technique. Participants reported a slight increase in task load when eye tracking was used. This could be due to the fact that the visualizations are changing faster when reacting to the user's eye movements instead of head movements.

With respect to conducted head movements, the results suggest that the class of AR glasses was more important than the applied guidance technique. As could be expected, participants conducted most head movements using pick-by-light as this was the only guidance where it was necessary to find the correct target by searching the environment. Using any pick-by-vision guidance reduced head movements. However, the techniques applied on the HoloLens had a stronger effect. For the DynSWave guidance, equipping the glasses with eye tracking lead to a minimal, non-significant improvement.

In contrast to the possible problems reported in 2.1, in our scenario we could not observe disadvantages arising from the central FOV of the HoloLens. Neither speed nor task load seem to be negatively affected in comparison to peripheral AR or picking-by-light. Regarding user acceptance, there was an indication that classic nonAR guidance is preferred over pick-by-vision using peripheral AR devices. However, guiding techniques applied on central FOV AR devices like the HoloLens were much more accepted by the participants. 
Eye tracking did not lead to significant differences in comparison to head-movement-based guidance. There are small hints that eye tracking might lead to faster task performance and less head movements if task load could be further reduced. This might be accomplished by a longer training phase.

\section{Conclusion}

In the scenario of order-picking, different AR pick-by-vision guidance techniques were evaluated on either a monocular peripheral FOV AR device and on binocular central FOV glasses and compared to non-AR picking-by-light. As examples for these two classes of AR devices, Google Glass and Microsoft HoloLens were chosen. Eye tracking was added in order to find out if such an addition can further improve guidance. The evaluation was done using a VR simulation framework for AR devices excluding interfering factors like tracking stability and guaranteeing high reproducibility.

The results reveal that AR pick-by-vision guidance could increase task performance and reduce task load when applied on HoloLens as central FOV AR device. The best performance could be achieved when applying the proposed DynSWave guidance on the HoloLens. This approach was also preferred by study participants. Overall, it is a promising alternative to the classic arrowbased guidance. Using the peripheral AR Google Glass, participants performed slower and reported a high task load, especially for the arrow-based guidance. Still, at least head movements could be reduced. Thus, using any of the pick-by-vision guidances can reduce physical effort. The results regarding the advantage of AR solutions over pick-by-light partly replicate previous work [7], but provide a more differentiated view on the two classes of AR devices and particular guiding techniques.

A validation of the results found in our simulated AR environment in a real-world scenario is an open task. The congruency of parts of the results with previous work conducting studies in the real world, however, supports our approach. Based on the presented findings, we are able to reduce the number of alternatives to be tested in the real-world scenario, which decreases implementation efforts (e.g. deploying solutions for different platforms), hardware modifications (attaching eye trackers to different AR glasses) and general the complexity of the real-world study design.

\section{ACKNOWLEDGEMENTS}

This research was partly supported by the Cluster of Excellence Cognitive Interaction Technology 'CITEC' (EXC 277) at Bielefeld University, which is funded by the German Research Foundation (DFG), and partly by the BMBF project "Adaptive and Mobile Action Assistance in Daily Living Activities" (ADAMAAS).

\section{References}

[1] G. Alce, K. Hermodsson, M. Wallergrd, L. Thern, and T. Hadzovic. A Prototyping Method to Simulate Wearable Augmented Reality Interaction in a Virtual Environment - A Pilot Study. International Journal of Virtual World and Human Computer Interaction, 2015.

[2] P. Baudisch and R. Rosenholtz. Halo: A technique for visualizing offscreen objects. In Proceedings of the SIGCHI Conference on Human Factors in Computing Systems, CHI '03, pages 481-488, New York, USA, 2003. ACM.

[3] F. Biocca, A. Tang, C. Owen, and F. Xiao. Attention Funnel: Omnidirectional 3d Cursor for Mobile Augmented Reality Platforms. In Proceedings of the SIGCHI Conference on Human Factors in Computing Systems, CHI '06, pages 1115-1122, New York, NY, USA, 2006. ACM.

[4] O. Cakmakci and J. Rolland. Head-worn displays: a review. Journal of Display Technology, 2(3):199-216, Sept. 2006.

[5] S. Feiner, B. MacIntyre, T. Höllerer, and A. Webster. A touring machine: Prototyping 3d mobile augmented reality systems for exploring the urban environment. Personal Technologies, 1(4):208-217, 1997.
[6] S. Feiner, B. Macintyre, and D. Seligmann. Knowledge-based Augmented Reality. Commun. ACM, 36(7):53-62, July 1993.

[7] A. Guo, S. Raghu, X. Xie, S. Ismail, X. Luo, J. Simoneau, S. Gilliland, H. Baumann, C. Southern, and T. Starner. A Comparison of Order Picking Assisted by Head-up Display (HUD), Cart-mounted Display (CMD), Light, and Paper Pick List. In Proceedings of the 2014 ACM International Symposium on Wearable Computers, ISWC '14, pages 71-78, New York, NY, USA, 2014. ACM.

[8] S. G. Hart and L. E. Staveland. Development of nasa-tlx (task load index): Results of empirical and theoretical research. Advances in psychology, 52:139-183, 1988.

[9] S. Henderson and S. Feiner. Exploring the Benefits of Augmented Reality Documentation for Maintenance and Repair. IEEE Transactions on Visualization and Computer Graphics, 17(10):1355-1368, 2011.

[10] N. Kishishita, K. Kiyokawa, J. Orlosky, T. Mashita, H. Takemura, and E. Kruijff. Analysing the effects of a wide field of view augmented reality display on search performance in divided attention tasks. In 2014 IEEE International Symposium on Mixed and Augmented Reality (ISMAR), pages 177-186, Sept. 2014.

[11] M. I. Nikolic and N. B. Sarter. Peripheral Visual Feedback: A Powerful Means of Supporting Effective Attention Allocation in EventDriven, Data-Rich Environments. Human Factors: The Journal of the Human Factors and Ergonomics Society, 43(1):30-38, Jan. 2001.

[12] E. Ragan, C. Wilkes, D. A. Bowman, and T. Hollerer. Simulation of augmented reality systems in purely virtual environments. In Virtual Reality Conference, 2009. VR 2009. IEEE, pages 287-288. IEEE, 2009.

[13] R. Reif and W. A. Günthner. Pick-by-vision: augmented reality supported order picking. The Visual Computer, 25(5-7):461-467, Mar. 2009.

[14] P. Renner and T. Pfeiffer. Attention Guiding Techniques using Peripheral Vision and Eye Tracking for Feedback in Augmented-Realitybased Assistance Systems. In 2017 IEEE Symposium on 3D User Interfaces (3DUI). IEEE, 2017.

[15] P. Renner and T. Pfeiffer. Evaluation of Attention Guiding Techniques for Augmented Reality-based Assistance in Picking and Assembly Tasks. In Proceedings of the 22nd International Conference on Intelligent User Interfaces Companion. ACM, 2017.

[16] T. Schinke, N. Henze, and S. Boll. Visualization of off-screen objects in mobile augmented reality. In Proceedings of the 12th international conference on Human computer interaction with mobile devices and services, pages 313-316. ACM, 2010.

[17] B. Schwerdtfeger and G. Klinker. Supporting Order Picking with Augmented Reality. In Proceedings of the 7th IEEE/ACM International Symposium on Mixed and Augmented Reality, ISMAR '08, pages 9194, Washington, DC, USA, 2008. IEEE Computer Society.

[18] B. Schwerdtfeger, R. Reif, W. A. Gunthner, G. Klinker, D. Hamacher, L. Schega, I. Bockelmann, F. Doil, and J. Tumler. Pick-by-Vision: A first stress test. In 2009 8th IEEE International Symposium on Mixed and Augmented Reality, pages 115-124, Oct. 2009.

[19] E. Steindecker, R. Stelzer, and B. Saske. Requirements for Virtualization of AR Displays within VR Environments. In R. Shumaker and S. Lackey, editors, Virtual, Augmented and Mixed Reality. Designing and Developing Virtual and Augmented Environments, number 8525 in Lecture Notes in Computer Science, pages 105-116. Springer International Publishing, June 2014.

[20] M. Tonnis and G. Klinker. Effective control of a car driver's attention for visual and acoustic guidance towards the direction of imminent dangers. In Proceedings of the 5th IEEE and ACM International Symposium on Mixed and Augmented Reality, pages 13-22. IEEE Computer Society, 2006.

[21] A. M. Wafaa, N. D. Bonnefoy, E. Dubois, P. Torguet, and J. P. Jessel. Virtual Reality Simulation for Prototyping Augmented Reality. In International Symposium on Ubiquitous Virtual Reality, 2008. ISUVR 2008, pages 55-58, July 2008.

[22] X. S. Zheng, C. Foucault, P. Matos da Silva, S. Dasari, T. Yang, and S. Goose. Eye-Wearable Technology for Machine Maintenance: Effects of Display Position and Hands-free Operation. In Proceedings of the 33rd Annual ACM Conference on Human Factors in Computing Systems, CHI '15, pages 2125-2134, New York, USA, 2015. ACM. 